\title{
Revisiting the trajectory of medical students' empathy, and impact of gender, specialty preferences and nationality: a systematic review
}

Freja Allerelli Andersen ${ }^{1 *+}$ (D) Ann-Sofie Bering Johansen ${ }^{1 \dagger}$, Jens Søndergaard ${ }^{2}$, Christina Maar Andersen ${ }^{3 \dagger}$ and Elisabeth Assing Hvidt ${ }^{2,4+}$

\begin{abstract}
Background: Empathy allows a physician to understand the patient's situation and feelings and respond appropriately. Consequently, empathy gives rise to better diagnostics and clinical outcomes. This systematic review investigates the level of empathy among medical students across the number of educational years and how this level relates to gender, specialty preferences, and nationality.

Method: In accordance with the Preferred Reporting Items for Systematic Reviews and Meta-Analyses (PRISMA), the authors conducted a systematic search of studies published between February 2010 and March 2019 investigating the level of empathy among medical students. The databases PubMed, EMBASE, and PsycINFO were searched. Studies employing quantitative methodologies and published in English or Scandinavian language and examining medical students exclusively were included.

Results: Thirty studies were included of which 24 had a cross-sectional and 6 a longitudinal study design. In 14 studies, significantly lower levels of empathy were reported by increase in the number of educational years. The remaining 16 studies identified both higher, mixed and unchanged levels. In 18 out of 27 studies it was reported that females had higher empathy scores than males. Only three out of nine studies found an association between empathy scores and specialty preferences. Nine out of 30 studies reported a propensity towards lower mean empathy scores in non-Western compared to Western countries.

Conclusion: The results revealed equivocal findings concerning how the empathy level among medical students develops among medical students across numbers of educational years and how empathy levels are associated with gender, specialty preferences, and nationality. Future research might benefit from focusing on how students' empathy is displayed in clinical settings, e.g. in clinical encounters with patients, peers and other health professionals.
\end{abstract}

Keywords: Empathy, Medical students, Systematic review

\footnotetext{
* Correspondence: frejaallerelli@gmail.com

${ }^{\dagger}$ Freja Allerelli Andersen and Ann-Sofie Bering Johansen contributed equally

to this work and join the first authorship.

${ }^{+}$Christina Maar Andersen and Elisabeth Assing Hvidt contributed equally to this work and join the last authorship.

${ }^{1}$ University of Southern Denmark, Campusvej 55, 5230 Odense M, Denmark

Full list of author information is available at the end of the article
}

(c) The Author(s). 2020 Open Access This article is distributed under the terms of the Creative Commons Attribution 4.0 International License (http://creativecommons.org/licenses/by/4.0/), which permits unrestricted use, distribution, and reproduction in any medium, provided you give appropriate credit to the original author(s) and the source, provide a link to the Creative Commons license, and indicate if changes were made. The Creative Commons Public Domain Dedication waiver (http://creativecommons.org/publicdomain/zero/1.0/) applies to the data made available in this article, unless otherwise stated. 


\section{Background}

Empathy is usually categorised as either affective (emotional), cognitive or a combination of both. The essence of affective empathy is compassion and the ability to enter into other peoples' feelings (Einfühlung). Cognitive empathy is described as "the ability to understand someone's situation without making it one's own" [1]. In the clinical setting and within the context of the patient-physician relationship, it is predominantly the cognitive empathy type that is valued and strived for. Mercer and Reynolds [2] define clinical empathy as the ability to a) understand the patient's situation, perspective and feelings (and their attached meanings); b) to communicate that understanding and check its accuracy and c) to act on that understanding with the patient in a helpful (therapeutic) way. An empathic physician is able to sense the patient's feelings while at the same time sustaining his or her professionalism [3]. Empathy has been shown to contribute substantially to building and maintaining a good patient-physician relationship [4]. Studies on empathy among general practitioners (physicians specialised in general practice) concluded that a general practitioner's display of empathy creates a relationship built on trust, openness, and safety and that a general practitioner's empathic attitude makes the patient feel supported and listened to $[5,6]$. Consequently, patients are more likely to disclose accurate and important information about themselves resulting in better diagnostics and clinical outcomes [7-9]. Steinhausen et al. [8] found that patients who rated their physician as having "high physician empathy" using the Consultation-and-Relational-Empathy (CARE) measure had a 20-fold higher probability of a better self-reported medical treatment outcome compared to patients who rated their physician to have "low physician empathy." Furthermore, studying patients with diabetes, Hojat et al. [9] found a strong correlation between an empathic physician (measured through the Jefferson Scale of Physician Empathy (JSPE)) and lower values of lipoprotein cholesterol (LDL) and glycosylated haemoglobin (HbA1c). Beyond clinical outcomes, empathic communication has been shown to enhance patient satisfaction, compliance and patient empowerment [10-12]. Additionally, regarding physicianrelated benefits of empathy, physicians who perceive themselves as being empathic experience empathy as a source of professional satisfaction and meaningfulness protecting against burn-out $[5,13,14]$. As an offshoot of the large body of research documenting the beneficial effects of physician empathy, empathy development among medical students has become a comprehensive research topic. Moreover, the association between levels of empathy among medical students and variables such as gender, nationality and/or specialty preferences has received an increased focus among researchers. Hojat et al. [15] found that medical students interested in primary care specialties had higher empathy scores than students showing interests in technology and procedure orientated specialties. Female and male physicians are furthermore shown to approach the patient-physician relationship differently [16]. For example, female physicians value psychosocial factors more than male physicians and engage to a larger extent in patient-centred and/or relationship-centred communication [17]. These varying cultural, social and psychological influences on empathy levels are also reflected in the fact that findings from studies conducted in different countries vary to a high degree $[18,19]$. Several research studies using student self-report measures to measure empathy levels have documented that a significant decline in empathy occurs among medical students as their training progresses $[20,21]$. Contrary to these finding, however, other studies have shown that empathy levels among medical students increase or that they are maintained [22-24]. Neumann et al. [25] published a systematic review on student empathy in 2011, concluding on the basis of 18 studies that empathy levels decline during medical education due to, mainly, an increase in student-patient contact and interaction. Colliver et al. [26] however, conducting a metaanalysis a year earlier, concluded that student empathy levels only decrease to a minimal degree if at all. Since then, more studies on the subject have been published that assumingly reflect all the new educational initiatives taking in relation to the medical curriculum that have empathy cultivation and preservation as a key goal, such as accompanying patients on medical visits making home visits, and reading medically related literature and poetry (narrative medicine) [27, 28]. Summarising the above, empathy is an important concept in health care and within educational research. However, as a consequence of many different definitions and understandings of empathy, and of different ways of measuring empathy, research in the area has also led to ambiguous results. There is therefore a need for an updated overview and review of the most recent research evidence regarding empathy among medical students.

The aim of this study was to perform a systematic review in accordance with the Preferred Reporting Items for Systematic Reviews and Meta-Analyses Guidelines (PRISMA) [29] of the literature published between February 2010 and March 2019. We sought to answer the following questions:

1. What are the empathy levels among medical students across the number of educational years?

2. How do levels of empathy relate to gender, specialty preferences, and nationality?

\section{Method}

\section{Search strategy}

The review was conducted according to the PRISMA guidelines [29]. AJ and FA conducted a systematic search in March 2019 informed by the research questions. Three 
databases were searched: PubMed, EMBASE, and PsycINFO. The following search words were used: 'empathy' AND 'medical student' AND ('decrease' OR 'increase'). Additionally, synonyms, the National Library of Medicine's Medical Subject Heading terms (MeSH) and subject headings were identified and applied (see Additional file 1). During the full-text screening, we also performed a manual search of reference sections to identify studies not found through the database searches.

\section{Inclusion and exclusion criteria}

Inclusion criteria were the following:

- Studies published between February 2010 and March 2019

- Quantitative studies

- Studies in English or Scandinavian language

- Study population restricted to medical students

Exclusion criteria were the following:

- Qualitative studies

- Intervention studies

- Psychometric studies

- Conference abstracts

- Non-empirical texts

\section{Selection of data}

Titles and abstracts of the studies were screened. In the case of uncertainty, full texts were read. Disagreement between reviewers (AJ and FA) regarding inclusion of the studies was settled through discussion until concordance was reached. Afterwards, AJ and FA read the full texts of the eligible studies. Together, the authors summarised and analysed the methods, results, and discussion sections of the studies. Independently, we applied methodological quality assessment tools on the different studies according to study design. Crombie's items [30] were applied to cross-sectional studies $(n=24)$ and consist of seven items rated as "yes" ( 1 point), "unclear" ( 0.5 points) and "no" ( 0 points), with a maximum of 7 points. The quality of the longitudinal studies $(n=6)$ was assessed by employing a structured 33-point checklist from Tooth et al. (see Additional file 2) [31]. Possible disagreements were discussed and settled and there was inter-rater reliability.

\section{Results}

\section{Included studies}

The search resulted in 1501 studies, of which 347 were duplicates (see Fig. 1). A total of 1154 studies were screened by title and abstract. Among these, 41 studies were selected for full-text reading since they fulfilled the inclusion criteria. During full-text reading, reference sections were also screened, which revealed another 12 eligible studies. A total of 53 studies were full-text screened. We excluded 23 of the 53 studies since they did not apply to our aim $(n=20)$ or were in a language other than English or Scandinavian $(n=3)$. Altogether 30 studies were included in the review.

\section{Study characteristics \\ Study design and sample sizes}

Of the 30 studies included in the review, 24 studies were cross-sectional and 6 studies longitudinal (see study characteristics and main findings in Table 1). Sample sizes of the cross-sectional studies varied from 129 [28] to 5521 [48] participants. In the longitudinal studies, sample size varied from 72 [52] to 1653 [55] participants.

\section{Scales}

All cross-sectional studies employed the Jefferson Scale of Physician Empathy student version (JSPE-S), except for four studies using the following scales: the Basic Empathy Scale [40], Measure of Patient-Centered Communication (MPCC) [28], Reading the Mind in the Eyes (RMET) and Balanced Emotional Empathy Scale (BEES) [37], and Empathic Skill Scale Form B and Conflict Tendency Scale [23].

All longitudinal studies used JSPE-S except for one that applied the Interpersonal Reactivity Index scale (IRI) [55]. One longitudinal study applied both an observational Objective Structured Clinical Examinations (OSCE) evaluation and JSPE-S [38]. Likewise, a crosssectional study used the Measure of Patient-Centered Communication (MPCC), which is also an observational scale that measures empathy [28].

\section{Country}

The studies were conducted in 20 different countries.

The Western countries were Australia [24], Belgium [40], New Zealand [42, 52], Portugal [32], USA [28, 35, 38, 56], United Kingdom [42, 49, 55].

The non-Western countries were Brazil [43], China [44, 45], Colombia [41, 51], Dominican Republic [41], Ecuador [51], Ethiopia [37], India [36, 39], Iran [21, 46, 47, 50], Korea [48], Kuwait [53], Malaysia [54], Pakistan [33], Trinidad and Tobago [34] and Turkey [23].

\section{Quality assessment and risk of bias in the included studies}

The quality assessment tools were used to identify the risk of bias. All included studies employed self-reporting questionnaires. Consequently, reporting bias was present which may have influenced the results. Three studies used small sample sizes, including respectively 129 [28], 77 [32], and 122 [56] study participants Hence, the findings of those studies may not be representative of the student population measured and it might over- and/or underestimate the outcome measures. 


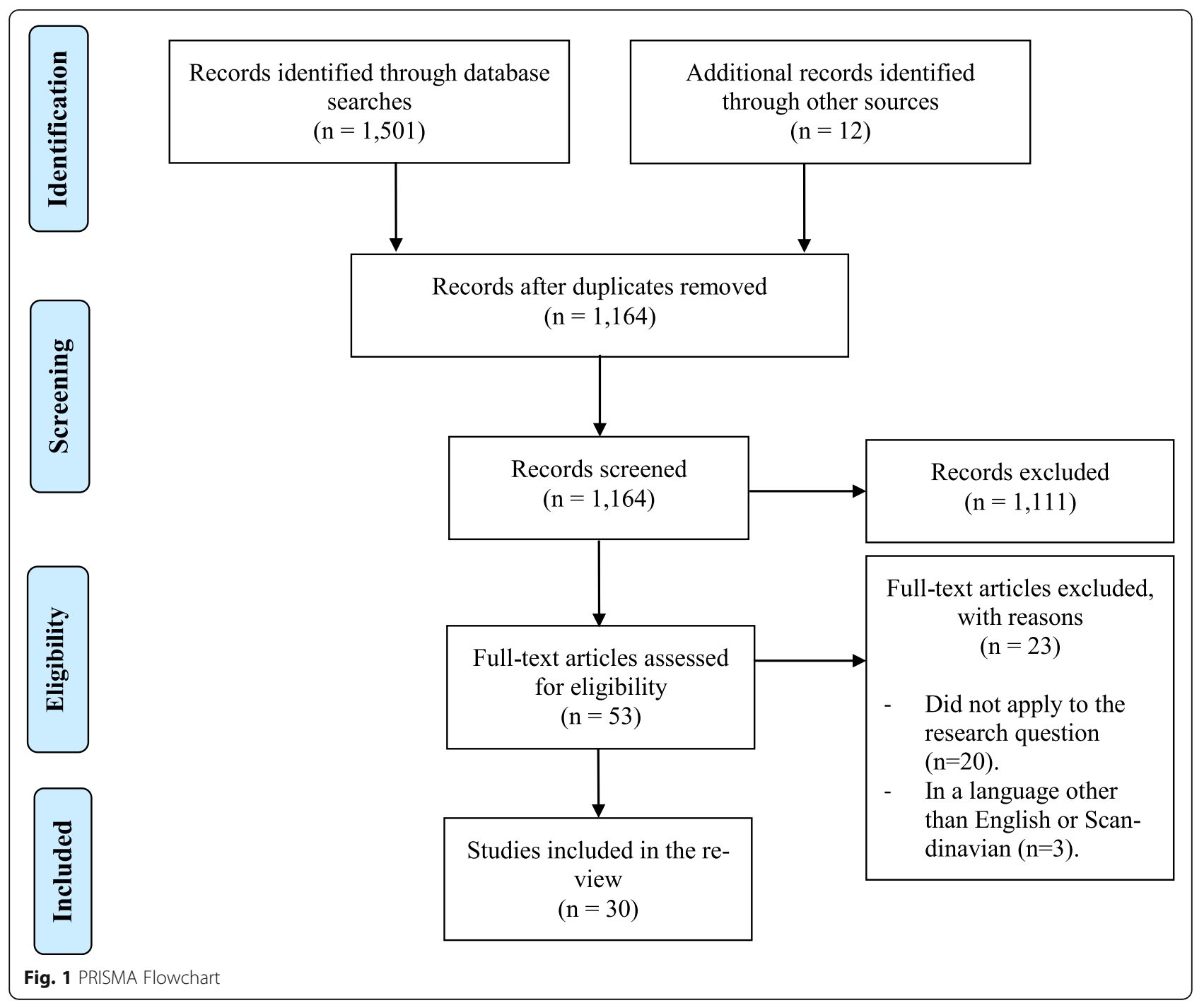

Out of 30 studies, 24 were single-institution studies $[21,23,24,27,28,32,34-40,43-46,49,50,52-56]$ making the results of these studies less generalisable and hereby affecting the studies' external validity.

One obvious limitation of the cross-sectional studies design was their inability to report changes over time. On the contrary, longitudinal studies could describe changes over time. Only one study used a control group of non-medical students, increasing its quality since it enabled comparison.

All studies, except for one [37], employed validated scales to examine the level of empathy. One study [53] employed the English validated JPSE-S on students who did not have English as their native language.

\section{The levels of empathy across number of educational years}

Significantly lower levels of empathy by increase in number of educational years were found in 14 out of 30 studies. Of these, 12 were cross-sectional studies [21, 33, 34,
$39-41,45-48,50,52,54]$ and two were longitudinal [52, 56]. All except one [55] of the cross-sectional studies used JSPE-S. Four cross-sectional studies [23, 27, 28, 44] reported a higher level of empathy among medical students at a higher year of medical school. Five crosssectional studies [24, 42, 43, 49,51] and one longitudinal study [32] found no statistically significant difference in empathy scores across the different years of medical education. Hasan et al. [53] reported higher empathy scores with higher educational years up until the fourth year, where a decreasing trend was observed. A crosssectional study [37] differentiated between emotional and cognitive empathy and found a higher cognitive empathy level in final year students compared to first-year students. On the contrary, a longitudinal study [55] found no change in cognitive empathy.

Chen et al. [38] conducted a longitudinal study, applying both self-administered empathy measures and observed empathy in an OSCE. It showed higher self-administered 
Table 1 Results

\begin{tabular}{|c|c|c|c|c|c|c|c|c|}
\hline Title & $\begin{array}{l}\text { Author, year } \\
\text { published }\end{array}$ & Country & Study objective & $\begin{array}{l}\text { Study } \\
\text { design }\end{array}$ & $\begin{array}{l}\text { Sample size } \\
\text { (response rate) }\end{array}$ & Scales $^{1}$ & Results & $\begin{array}{l}\text { Comments on } \\
\text { study quality }\end{array}$ \\
\hline $\begin{array}{l}\text { A latent growth } \\
\text { model suggests } \\
\text { that empathy of } \\
\text { medical students } \\
\text { does not decline } \\
\text { over time [32] }\end{array}$ & $\begin{array}{l}\text { Costa et al. } 2013 \\
\text { [32] }\end{array}$ & Portugal & $\begin{array}{l}\text { Investigate } \\
\text { empathy } \\
\text { throughout } \\
\text { medical school }\end{array}$ & Longitudinal & 77 & $\begin{array}{l}\text { JSPE-S } \\
\text { NEO-Five- } \\
\text { Factor } \\
\text { Inventory }\end{array}$ & $\begin{array}{l}\text { - Non-significant } \\
\text { positive evolu- } \\
\text { tion in em- } \\
\text { pathy scores } \\
\text { across three } \\
\text { time points in } \\
\text { Portuguese } \\
\text { undergraduate } \\
\text { medical stu- } \\
\text { dents that } \\
\text { cover the pre- } \\
\text { clinical/clinical } \\
\text { transmission - } \\
\text { A significant } \\
\text { decline in em- } \\
\text { pathy was } \\
\text { found in fe- } \\
\text { males in the } \\
\text { transition } \\
\text { period from } \\
\text { pre-clinical to } \\
\text { the clinical } \\
\text { phase of } \\
\text { education }\end{array}$ & $\begin{array}{l}\text { - Single- } \\
\text { institution } \\
\text { survey - } \\
\text { Small sample } \\
\text { size }\end{array}$ \\
\hline $\begin{array}{l}\text { A Quantitative } \\
\text { Study of } \\
\text { Empathy in } \\
\text { Pakistani Medical } \\
\text { Students: } \\
\text { A Multicentered } \\
\text { Approach [33] }\end{array}$ & $\begin{array}{l}\text { Tariq et al. } 2017 \\
\text { [33] }\end{array}$ & Pakistan & $\begin{array}{l}\text { Investigate } \\
\text { empathy among } \\
\text { medical } \\
\text { students in the } \\
\text { context of } \\
\text { patient care }\end{array}$ & $\begin{array}{l}\text { Cross- } \\
\text { sectional }\end{array}$ & 1453 & JSPE-S & $\begin{array}{l}\text { - A statistically } \\
\text { significant } \\
\text { difference in } \\
\text { JSPE scores for } \\
\text { the } 5 \text { medical } \\
\text { year groups - } \\
\text { No significant } \\
\text { difference in } \\
\text { the JSPE scores } \\
\text { for males and } \\
\text { females - No } \\
\text { significant } \\
\text { difference in } \\
\text { the JSPE scores } \\
\text { across specialty } \\
\text { preference }\end{array}$ & $\begin{array}{c}\text { - Multi-center } \\
\text { survey - Re- } \\
\text { sponse rate } \\
\text { unspecified }\end{array}$ \\
\hline $\begin{array}{l}\text { An exploration } \\
\text { of changes in } \\
\text { cognitive and } \\
\text { emotional } \\
\text { empathy among } \\
\text { medical students } \\
\text { in the Caribbean } \\
\text { [34] }\end{array}$ & $\begin{array}{l}\text { Youssef et al. } 2014 \\
{[34]}\end{array}$ & $\begin{array}{l}\text { Trinidad } \\
\text { \&Tobago }\end{array}$ & $\begin{array}{l}\text { Investigate the } \\
\text { empathy profile } \\
\text { across all five } \\
\text { years of training } \\
\text { in a medical } \\
\text { school }\end{array}$ & $\begin{array}{l}\text { Cross- } \\
\text { sectional }\end{array}$ & $667(67 \%)$ & $\begin{array}{l}\text { JSPE-STEQ } \\
\text { RMET }\end{array}$ & $\begin{array}{l}\text { - Highest scores } \\
\text { were observed } \\
\text { when students } \\
\text { enter medical } \\
\text { school and the } \\
\text { lowest scores } \\
\text { among third- } \\
\text { year students - } \\
\text { Female mean } \\
\text { scores were } \\
\text { significantly } \\
\text { higher than } \\
\text { male - No sig- } \\
\text { nificant effects } \\
\text { of ethnicity or } \\
\text { specialisation }\end{array}$ & $\begin{array}{l}\text { - Single- } \\
\text { institution } \\
\text { survey - } \\
\text { Highest re- } \\
\text { sponse rate } \\
\text { at year one }\end{array}$ \\
\hline $\begin{array}{l}\text { Characterizing } \\
\text { changes in } \\
\text { student } \\
\text { empathy } \\
\text { throughout } \\
\text { medical school } \\
\text { [35] }\end{array}$ & $\begin{array}{l}\text { Chen et al. } 2012 \\
\text { [35] }\end{array}$ & USA & $\begin{array}{l}\text { Investigate long- } \\
\text { term trajectories } \\
\text { of empathy } \\
\text { among medical } \\
\text { students }\end{array}$ & Longitudinal & 1162 (81\%) & JSPE-S & $\begin{array}{l}\text { - Empathy levels } \\
\text { increased from } \\
\text { the beginning } \\
\text { of medical } \\
\text { school until } \\
\text { the end of } \\
\text { preclinical } \\
\text { years, followed } \\
\text { by a decline in } \\
\text { the third year }\end{array}$ & $\begin{array}{l}\text { - Single- } \\
\text { institution } \\
\text { survey - } \\
\text { Varying re- } \\
\text { sponse rate } \\
(54-99 \%)\end{array}$ \\
\hline
\end{tabular}


Table 1 Results (Continued)

\begin{tabular}{|c|c|c|c|c|c|c|c|c|}
\hline Title & $\begin{array}{l}\text { Author, year } \\
\text { published }\end{array}$ & Country & Study objective & $\begin{array}{l}\text { Study } \\
\text { design }\end{array}$ & $\begin{array}{l}\text { Sample size } \\
\text { (response rate) }\end{array}$ & Scales $^{1}$ & Results & $\begin{array}{l}\text { Comments on } \\
\text { study quality }\end{array}$ \\
\hline & & & & & & & $\begin{array}{l}\text { of medical } \\
\text { school that } \\
\text { persisted } \\
\text { throughout } \\
\text { graduation - } \\
\text { Females } \\
\text { showed } \\
\text { significantly } \\
\text { higher } \\
\text { empathy levels } \\
\text { than males }\end{array}$ & \\
\hline $\begin{array}{l}\text { Clinical empathy } \\
\text { in medical } \\
\text { students in India } \\
\text { measured using } \\
\text { the Jefferson } \\
\text { Scale of } \\
\text { Empathy- } \\
\text { Student Version } \\
\text { [36] }\end{array}$ & $\begin{array}{l}\text { Chatterjee et al. } \\
2017\end{array}$ & India & $\begin{array}{l}\text { Investigate } \\
\text { clinical empathy } \\
\text { and the various } \\
\text { associated } \\
\text { factors in } \\
\text { medical } \\
\text { students } \\
\text { spanning } 4 \text { years } \\
\text { of } \\
\text { undergraduate } \\
\text { study }\end{array}$ & $\begin{array}{l}\text { Cross- } \\
\text { sectional }\end{array}$ & 418 (69.7\%) & JSPE-S & $\begin{array}{l}\text { - Mean empathy } \\
\text { scores fell from } \\
\text { the first to the } \\
\text { third semester, } \\
\text { then more or } \\
\text { less plateaued, } \\
\text { and then rose } \\
\text { again in the } \\
\text { seventh } \\
\text { semester - } \\
\text { Females } \\
\text { having } \\
\text { significantly } \\
\text { higher } \\
\text { empathy } \\
\text { scores than } \\
\text { males }\end{array}$ & $\begin{array}{l}\text { - Single- } \\
\text { institution } \\
\text { survey }\end{array}$ \\
\hline $\begin{array}{l}\text { Comparative } \\
\text { cross-sectional } \\
\text { study of em- } \\
\text { pathy among } \\
\text { first year and } \\
\text { final year med- } \\
\text { ical students in } \\
\text { Jimma Univer- } \\
\text { sity, Ethiopia: } \\
\text { Steady state of } \\
\text { the heart and } \\
\text { opening of the } \\
\text { eyes [37] }\end{array}$ & $\begin{array}{l}\text { Dehning, S. et al. } \\
2012\end{array}$ & Ethiopia & $\begin{array}{l}\text { Investigate } \\
\text { whether } \\
\text { empathy } \\
\text { increases with } \\
\text { medical training } \\
\text { and identify the } \\
\text { socio- } \\
\text { demographic } \\
\text { background of } \\
\text { medical stu- } \\
\text { dents influen- } \\
\text { cing their } \\
\text { empathy levels }\end{array}$ & $\begin{array}{l}\text { Cross- } \\
\text { sectional }\end{array}$ & 237 & $\begin{array}{l}\text { BEES } \\
\text { RMET }\end{array}$ & $\begin{array}{l}\text { - No significant } \\
\text { difference in } \\
\text { emotional } \\
\text { empathy } \\
\text { between first- } \\
\text { year and final } \\
\text { year medical } \\
\text { students - } \\
\text { Higher cogni- } \\
\text { tive empathy } \\
\text { among final } \\
\text { year students } \\
\text { as compared } \\
\text { to first-year } \\
\text { students - } \\
\text { Males scored } \\
\text { lower mean } \\
\text { cognitive and } \\
\text { emotional em- } \\
\text { pathy scores } \\
\text { than females }\end{array}$ & $\begin{array}{l}\text { - Single- } \\
\text { institution } \\
\text { survey - No } \\
\text { response } \\
\text { rate - Instru- } \\
\text { ments not } \\
\text { validated }\end{array}$ \\
\hline $\begin{array}{l}\text { Comparing a } \\
\text { Self- } \\
\text { Administered } \\
\text { Measure of Em- } \\
\text { pathy with Ob- } \\
\text { served Behaviour } \\
\text { Among Medical } \\
\text { Students [38] }\end{array}$ & Chen et al. 2010 & USA & $\begin{array}{l}\text { Investigate the } \\
\text { relationship } \\
\text { between self- } \\
\text { administered, } \\
\text { JSPE-S, and ob- } \\
\text { served empathy }\end{array}$ & Longitudinal & $\begin{array}{l}\text { 2nd year: } 163 \\
\text { (97.6\%) 3rd year: } \\
159(98.1 \%)\end{array}$ & $\begin{array}{l}\text { JSPE- } \\
\text { SOSCE- } \\
\text { evaluation }\end{array}$ & $\begin{array}{l}\text { - Second-year } \\
\text { students had } \\
\text { higher JSPE- } \\
\text { scores com- } \\
\text { pared to third- } \\
\text { year students, } \\
\text { but the aver- } \\
\text { age observed } \\
\text { empathy score } \\
\text { for second-year } \\
\text { students was } \\
\text { lower than the } \\
\text { observed em- } \\
\text { pathy scores } \\
\text { for third-year } \\
\text { students - A } \\
\text { trend towards }\end{array}$ & $\begin{array}{l}\text { - Single- } \\
\text { institution } \\
\text { survey - A } \\
\text { small num- } \\
\text { ber of OSCE } \\
\text { participants }\end{array}$ \\
\hline
\end{tabular}


Table 1 Results (Continued)

\begin{tabular}{|c|c|c|c|c|c|c|c|c|}
\hline Title & $\begin{array}{l}\text { Author, year } \\
\text { published }\end{array}$ & Country & Study objective & $\begin{array}{l}\text { Study } \\
\text { design }\end{array}$ & $\begin{array}{l}\text { Sample size } \\
\text { (response rate) }\end{array}$ & Scales $^{1}$ & Results & $\begin{array}{l}\text { Comments on } \\
\text { study quality }\end{array}$ \\
\hline & & & & & & & $\begin{array}{l}\text { a decline in } \\
\text { measured em- } \\
\text { pathy with in- } \\
\text { creased clinical } \\
\text { training with a } \\
\text { self- } \\
\text { administered } \\
\text { instrument, but } \\
\text { an improve- } \\
\text { ment in ob- } \\
\text { served em- } \\
\text { pathy among } \\
\text { those clinically } \\
\text { experienced } \\
\text { (i.e. third-year- } \\
\text { students) }\end{array}$ & \\
\hline $\begin{array}{l}\text { Comparison of } \\
\text { empathy score } \\
\text { among medical } \\
\text { students in both } \\
\text { basic and clinical } \\
\text { levels [21] }\end{array}$ & $\begin{array}{l}\text { Khademalhosseini } \\
\text { et al. } 2014\end{array}$ & Iran & $\begin{array}{l}\text { Investigate and } \\
\text { compare the } \\
\text { empathy scores } \\
\text { of medical } \\
\text { students } \\
\text { between basic } \\
\text { sciences and } \\
\text { clinical levels }\end{array}$ & $\begin{array}{l}\text { Cross- } \\
\text { sectional }\end{array}$ & 260 & JSPE-S & $\begin{array}{l}\text { - Empathy } \\
\text { scores among } \\
\text { medical } \\
\text { students } \\
\text { decreased by } \\
\text { the increase in } \\
\text { their } \\
\text { educational } \\
\text { years - Females } \\
\text { had higher } \\
\text { mean empathy } \\
\text { students than } \\
\text { males }\end{array}$ & $\begin{array}{l}\text { - Single- } \\
\text { institution } \\
\text { survey - Re- } \\
\text { sponse rate } \\
\text { unspecified }\end{array}$ \\
\hline $\begin{array}{l}\text { Comparison of } \\
\text { Empathy Skills } \\
\text { and Conflict } \\
\text { Tendency in } \\
\text { Preclinical and } \\
\text { Clinical Phase } \\
\text { Turkish Medical } \\
\text { Students: a } \\
\text { Cross-Sectional } \\
\text { Study [23] }\end{array}$ & Atay et al. 2014 & Turkey & $\begin{array}{l}\text { Investigate the } \\
\text { differences and } \\
\text { relations of } \\
\text { empathy and } \\
\text { conflict } \\
\text { tendencies of } \\
\text { medical } \\
\text { students in } \\
\text { preclinical and } \\
\text { clinical phase }\end{array}$ & $\begin{array}{l}\text { Cross- } \\
\text { sectional }\end{array}$ & 186 (55\%) & $\begin{array}{l}\text { Empathic } \\
\text { Skill Scale } \\
\text { B Form } \\
\text { Conflict } \\
\text { Tendency } \\
\text { Scale }\end{array}$ & $\begin{array}{l}\text { - A significantly } \\
\text { higher score of } \\
\text { empathy skills } \\
\text { in last year } \\
\text { medical } \\
\text { students as } \\
\text { compared to } \\
\text { first-year and } \\
\text { fourth-year stu- } \\
\text { dents - No sig- } \\
\text { nificant differ- } \\
\text { ences in } \\
\text { empathy skill } \\
\text { scores among } \\
\text { males and } \\
\text { females }\end{array}$ & $\begin{array}{l}\text { - Single- } \\
\text { institution } \\
\text { survey - Low } \\
\text { response } \\
\text { rate }\end{array}$ \\
\hline $\begin{array}{l}\text { Cross sectional } \\
\text { assessment of } \\
\text { empathy among } \\
\text { undergraduates } \\
\text { from a medical } \\
\text { college [39] }\end{array}$ & $\begin{array}{l}\text { Shashikumar et al. } \\
2014\end{array}$ & India & $\begin{array}{l}\text { Investigate } \\
\text { empathy among } \\
\text { medical } \\
\text { students of } \\
\text { various years }\end{array}$ & $\begin{array}{l}\text { Cross- } \\
\text { sectional }\end{array}$ & 488 (75\%) & JSPE-S & $\begin{array}{l}\text { - Empathy } \\
\text { declines during } \\
\text { medical school, } \\
\text { but only } \\
\text { significant } \\
\text { when } \\
\text { comparing } \\
\text { first-year and } \\
\text { seventh-year- } \\
\text { Significantly } \\
\text { higher em- } \\
\text { pathy score in } \\
\text { females com- } \\
\text { pared to males } \\
\text { - No significant } \\
\text { differences in } \\
\text { empathy } \\
\text { scores among } \\
\text { different } \\
\text { groups of spe- } \\
\text { cialty }\end{array}$ & $\begin{array}{l}\text { - Single- } \\
\text { institution } \\
\text { survey - A } \\
\text { low number } \\
\text { of re- } \\
\text { sponders in } \\
\text { 7th semester }\end{array}$ \\
\hline
\end{tabular}


Table 1 Results (Continued)

\begin{tabular}{|c|c|c|c|c|c|c|c|c|}
\hline Title & $\begin{array}{l}\text { Author, year } \\
\text { published }\end{array}$ & Country & Study objective & $\begin{array}{l}\text { Study } \\
\text { design }\end{array}$ & $\begin{array}{l}\text { Sample size } \\
\text { (response rate) }\end{array}$ & Scales $^{1}$ & Results & $\begin{array}{l}\text { Comments on } \\
\text { study quality }\end{array}$ \\
\hline & & & & & & \multicolumn{3}{|c|}{ preferences } \\
\hline $\begin{array}{l}\text { Decline of } \\
\text { empathy among } \\
\text { medical } \\
\text { students: } \\
\text { Dehumanization } \\
\text { or useful coping } \\
\text { process? [40] }\end{array}$ & Triffaux et al. 2019 & Belgium & $\begin{array}{l}\text { Investigate } \\
\text { empathy } \\
\text { decline among } \\
\text { Belgian medical } \\
\text { students across } \\
\text { the different } \\
\text { years of } \\
\text { education and } \\
\text { compare the } \\
\text { level of } \\
\text { empathy with } \\
\text { commercial } \\
\text { students }\end{array}$ & $\begin{array}{l}\text { Cross- } \\
\text { sectional }\end{array}$ & $\begin{array}{l}1353 \text { medical } \\
\text { students and } \\
249 \text { commercial } \\
\text { students }\end{array}$ & $\begin{array}{l}\text { The Basic } \\
\text { Empathy } \\
\text { Scale }\end{array}$ & $\begin{array}{l}\text { - A significant } \\
\text { decline in } \\
\text { empathy } \\
\text { scores among } \\
\text { medical } \\
\text { students - } \\
\text { Significantly } \\
\text { higher } \\
\text { empathy } \\
\text { scores among } \\
\text { females in } \\
\text { comparison } \\
\text { with males - } \\
\text { Significant } \\
\text { higher } \\
\text { empathy } \\
\text { scores on all } \\
\text { aspects of } \\
\text { empathy for } \\
\text { medical } \\
\text { students } \\
\text { compared to } \\
\text { commercial } \\
\text { students }\end{array}$ & $\begin{array}{l}\text { - Single- } \\
\text { institution } \\
\text { survey - Un- } \\
\text { specified } \\
\text { response- } \\
\text { rate }\end{array}$ \\
\hline $\begin{array}{l}\text { Empathic } \\
\text { orientation } \\
\text { among medical } \\
\text { students from } \\
\text { three universities } \\
\text { in Barranquilla, } \\
\text { Colombia and } \\
\text { one university in } \\
\text { the Dominican } \\
\text { Republic [41] }\end{array}$ & $\begin{array}{l}\text { Diaz Narvaez et al. } \\
2014\end{array}$ & $\begin{array}{l}\text { Colombia } \\
\text { The } \\
\text { Dominican } \\
\text { Republic }\end{array}$ & $\begin{array}{l}\text { Investigate and } \\
\text { compare } \\
\text { empathic } \\
\text { orientation in } \\
\text { medical } \\
\text { students from } \\
\text { four universities }\end{array}$ & $\begin{array}{l}\text { Cross- } \\
\text { sectional }\end{array}$ & $\begin{array}{l}\text { Universidad del } \\
\text { Norte: } n=345 \\
(40.16 \%) \\
\text { Universidad San } \\
\text { Martín, } n=283 \\
(57.4 \%) \\
\text { Universidad } \\
\text { Libre, } n=695 \\
(61.56 \%) \text {. } \\
\text { Universidad } \\
\text { Central del Este, } \\
n=515(60.38 \%)\end{array}$ & JSPE-S & $\begin{array}{l}\text { - Empathy } \\
\text { values reduced } \\
\text { as courses } \\
\text { advanced in all } \\
\text { schools } \\
\text { analysed - No } \\
\text { differences } \\
\text { between } \\
\text { genders }\end{array}$ & $\begin{array}{l}\text { - Multi-center } \\
\text { survey - Lack } \\
\text { of } \\
\text { consistency } \\
\text { between text } \\
\text { and table } \\
\text { presentation } \\
\text { of results }\end{array}$ \\
\hline $\begin{array}{l}\text { Empathy among } \\
\text { undergraduate } \\
\text { medical } \\
\text { students: A } \\
\text { multi-centre } \\
\text { cross-sectional } \\
\text { comparison of } \\
\text { students begin- } \\
\text { ning and ap- } \\
\text { proaching the } \\
\text { end of their } \\
\text { course [42] }\end{array}$ & Quince et al. 2016 & $\begin{array}{l}\text { United } \\
\text { Kingdom } \\
\text { (UK) New } \\
\text { Zealand } \\
\text { (NZ) }\end{array}$ & $\begin{array}{l}\text { Investigate } \\
\text { empathy among } \\
\text { students at the } \\
\text { beginning and } \\
\text { end of } \\
\text { undergraduate } \\
\text { medical training } \\
\text { in multiple } \\
\text { medical schools }\end{array}$ & $\begin{array}{l}\text { Cross- } \\
\text { sectional }\end{array}$ & $\begin{array}{l}1139 \text { UK: } 1 \text { st/ } \\
\text { 2nd year: } 652 \\
\text { (54.9\%) Final } \\
\text { year: } 487(48.1) \\
\text { NZ 1st year: } 721 \\
\text { (24\%) Final year: } \\
476(15.2 \%)\end{array}$ & JSPE-SIRI & $\begin{array}{l}\text { - No significant } \\
\text { differences in } \\
\text { empathy } \\
\text { scores } \\
\text { between } \\
\text { students } \\
\text { starting and } \\
\text { approaching } \\
\text { the end of } \\
\text { their course - } \\
\text { Females had } \\
\text { significantly } \\
\text { higher } \\
\text { empathy } \\
\text { scores than } \\
\text { males }\end{array}$ & $\begin{array}{l}\text { - Multi-center } \\
\text { survey - A } \\
\text { low response } \\
\text { rate in NZ } \\
\text { final year }\end{array}$ \\
\hline $\begin{array}{l}\text { Empathy } \\
\text { differences by } \\
\text { gender and } \\
\text { specialty } \\
\text { preference in } \\
\text { medical } \\
\text { students: a study } \\
\text { in Brazil [43] }\end{array}$ & Santos et al. 2016 & Brazil & $\begin{array}{l}\text { Investigate } \\
\text { medical } \\
\text { students' } \\
\text { empathy and to } \\
\text { examine } \\
\text { empathy } \\
\text { differences by } \\
\text { students' socio- } \\
\text { demographic } \\
\text { characteristics, } \\
\text { including gen- } \\
\text { der, and }\end{array}$ & $\begin{array}{l}\text { Cross- } \\
\text { sectional }\end{array}$ & $226(70.6 \%)$ & JSPE-S & $\begin{array}{l}\text { - Consistently } \\
\text { high scores of } \\
\text { empathy in } \\
\text { medical } \\
\text { students } \\
\text { enrolled in all } \\
\text { levels of } \\
\text { training - } \\
\text { Higher } \\
\text { empathy } \\
\text { scores in } \\
\text { females and }\end{array}$ & $\begin{array}{l}\text { - Single- } \\
\text { institution } \\
\text { survey }\end{array}$ \\
\hline
\end{tabular}


Table 1 Results (Continued)

\begin{tabular}{|c|c|c|c|c|c|c|c|c|}
\hline Title & $\begin{array}{l}\text { Author, year } \\
\text { published }\end{array}$ & Country & Study objective & $\begin{array}{l}\text { Study } \\
\text { design }\end{array}$ & $\begin{array}{l}\text { Sample size } \\
\text { (response rate) }\end{array}$ & Scales $^{1}$ & Results & $\begin{array}{l}\text { Comments on } \\
\text { study quality }\end{array}$ \\
\hline & & & $\begin{array}{l}\text { specialty } \\
\text { preference }\end{array}$ & & & & $\begin{array}{l}\text { students who } \\
\text { intend to } \\
\text { pursue people- } \\
\text { oriented } \\
\text { specialties }\end{array}$ & \\
\hline $\begin{array}{l}\text { Empathy in } \\
\text { Chinese medical } \\
\text { students: } \\
\text { psychometric } \\
\text { characteristics } \\
\text { and differences } \\
\text { by gender and } \\
\text { year of medical } \\
\text { education [44] }\end{array}$ & Wen et al. 2013 & China & $\begin{array}{l}\text { Investigate the } \\
\text { psychometric } \\
\text { properties of } \\
\text { the JSPE-S } \\
\text { among a sam- } \\
\text { ple of Chinese } \\
\text { medical stu- } \\
\text { dents and inves- } \\
\text { tigate the } \\
\text { primary levels of } \\
\text { empathy of the } \\
\text { medical stu- } \\
\text { dents and ana- } \\
\text { lyse group } \\
\text { differences }\end{array}$ & $\begin{array}{l}\text { Cross- } \\
\text { sectional }\end{array}$ & 753 (83.5\%) & JSPE-S & $\begin{array}{l}\text { - Statistically } \\
\text { significant } \\
\text { differences in } \\
\text { empathy } \\
\text { scores among } \\
\text { medical } \\
\text { students in } \\
\text { different years } \\
\text { of medical } \\
\text { school. The } \\
\text { first-year stu- } \\
\text { dents had the } \\
\text { lowest em- } \\
\text { pathy scores } \\
\text { and the fourth- } \\
\text { year students } \\
\text { had the high- } \\
\text { est empathy } \\
\text { scores. - Signifi- } \\
\text { cant higher } \\
\text { empathy } \\
\text { scores among } \\
\text { females }\end{array}$ & $\begin{array}{l}\text { - Single- } \\
\text { institution } \\
\text { survey - Ap- } \\
\text { plied mean } \\
\text { data as a } \\
\text { substitution } \\
\text { for missing } \\
\text { data }\end{array}$ \\
\hline $\begin{array}{l}\text { Empathy in } \\
\text { Chinese eight- } \\
\text { year medical } \\
\text { program stu- } \\
\text { dents: differ- } \\
\text { ences by school } \\
\text { year, educational } \\
\text { stage, and future } \\
\text { career prefer- } \\
\text { ence [45] }\end{array}$ & Li et al. 2018 & China & $\begin{array}{l}\text { Investigate the } \\
\text { factor structure } \\
\text { of a Chinese } \\
\text { version of the } \\
\text { JSPE-S with } \\
\text { Chinese stu- } \\
\text { dents, as well as } \\
\text { to assess the dif- } \\
\text { ferences in em- } \\
\text { pathy scores }\end{array}$ & $\begin{array}{l}\text { Cross- } \\
\text { sectional }\end{array}$ & 442 (83\%) & JSPE-S & $\begin{array}{l}\text { - A statistically } \\
\text { significant } \\
\text { difference in } \\
\text { the mean JSPE- } \\
\text { S scores in dif- } \\
\text { ferent school } \\
\text { years. There } \\
\text { was a differ- } \\
\text { ence between } \\
\text { fifth- and sixth- } \\
\text { year students } \\
\text { (109.1 vs. } \\
\text { 101.2). The } \\
\text { seventh-year } \\
\text { students had } \\
\text { significant dif- } \\
\text { ferences in em- } \\
\text { pathy scores } \\
\text { when com- } \\
\text { pared to the } \\
\text { first-year stu- } \\
\text { dents ( } 99.5 \\
\text { vs.107.6) and } \\
\text { seventh-year } \\
\text { students dif- } \\
\text { fered from } \\
\text { fifth-year stu- } \\
\text { dents ( } 99.5 \text { vs. } \\
109.1) \text { - No sig- } \\
\text { nificant differ- } \\
\text { ences in } \\
\text { empathy } \\
\text { scores among } \\
\text { females and } \\
\text { males - A sta- } \\
\text { tistically signifi- } \\
\text { cant difference } \\
\text { in empathy }\end{array}$ & $\begin{array}{l}\text { - Single- } \\
\text { institution } \\
\text { survey - Par- } \\
\text { ticipants } \\
\text { among the } \\
\text { top level of } \\
\text { medical stu- } \\
\text { dents in } \\
\text { China }\end{array}$ \\
\hline
\end{tabular}


Table 1 Results (Continued)

\begin{tabular}{|c|c|c|c|c|c|c|c|c|}
\hline Title & $\begin{array}{l}\text { Author, year } \\
\text { published }\end{array}$ & Country & Study objective & $\begin{array}{l}\text { Study } \\
\text { design }\end{array}$ & $\begin{array}{l}\text { Sample size } \\
\text { (response rate) }\end{array}$ & Scales $^{1}$ & Results & $\begin{array}{l}\text { Comments on } \\
\text { study quality }\end{array}$ \\
\hline & & & & & & & $\begin{array}{l}\text { mean score ac- } \\
\text { cording to fu- } \\
\text { ture career } \\
\text { preference. } \\
\text { Students who } \\
\text { preferred not } \\
\text { to become } \\
\text { doctors had } \\
\text { lower empathy } \\
\text { than those } \\
\text { who did prefer } \\
\text { to become } \\
\text { doctors }\end{array}$ & \\
\hline $\begin{array}{l}\text { Empathy in } \\
\text { Iranian medical } \\
\text { students: A } \\
\text { comparison by } \\
\text { age, gender, } \\
\text { academic } \\
\text { performance } \\
\text { and specialty } \\
\text { preferences [46] }\end{array}$ & $\begin{array}{l}\text { Benabbas et al. } \\
2016\end{array}$ & Iran & $\begin{array}{l}\text { Investigate self- } \\
\text { reported em- } \\
\text { pathy and its al- } \\
\text { teration during } \\
\text { medical school } \\
\text { in Iranian med- } \\
\text { ical students }\end{array}$ & $\begin{array}{l}\text { Cross- } \\
\text { sectional }\end{array}$ & 459 (91.8\%) & JSPE-S & $\begin{array}{l}\text { - The mean } \\
\text { score of } \\
\text { empathy } \\
\text { among interns } \\
\text { was } \\
\text { significantly } \\
\text { lower than } \\
\text { both trainees } \\
\text { and preclinical } \\
\text { students - No } \\
\text { statistically } \\
\text { significant } \\
\text { differences in } \\
\text { empathy } \\
\text { scores } \\
\text { between } \\
\text { genders - } \\
\text { Empathy score } \\
\text { was not } \\
\text { related to } \\
\text { specialty } \\
\text { choice }\end{array}$ & $\begin{array}{l}\text { - Single- } \\
\text { institution } \\
\text { survey }\end{array}$ \\
\hline $\begin{array}{l}\text { Empathy in } \\
\text { Iranian medical } \\
\text { students: } \\
\text { Measurement } \\
\text { model of the } \\
\text { Jefferson Scale } \\
\text { of Empathy [47] }\end{array}$ & Shariat et al. 2013 & Iran & $\begin{array}{l}\text { Investigate } \\
\text { empathy in } \\
\text { Iranian medical } \\
\text { students, as well } \\
\text { as to determine } \\
\text { the } \\
\text { measurement } \\
\text { model and } \\
\text { psychometric } \\
\text { properties of JSE } \\
\text { in Iranian } \\
\text { students }\end{array}$ & $\begin{array}{l}\text { Cross- } \\
\text { sectional }\end{array}$ & $1187(76.7 \%)$ & JSPE-S & $\begin{array}{l}\text { - Significant } \\
\text { decreasing } \\
\text { linear trend in } \\
\text { the score of } \\
\text { empathy with } \\
\text { increasing } \\
\text { years of } \\
\text { education - } \\
\text { Females } \\
\text { outscored } \\
\text { male students } \\
\text { in empathy - } \\
\text { JSPE-S showed } \\
\text { acceptable in- } \\
\text { ternal } \\
\text { consistency } \\
\text { and re-test } \\
\text { reliability }\end{array}$ & $\begin{array}{l}\text { - Multi- } \\
\text { institution } \\
\text { survey - Vari- } \\
\text { able re- } \\
\text { sponse rates } \\
\text { at different } \\
\text { universities }\end{array}$ \\
\hline $\begin{array}{l}\text { Empathy in } \\
\text { Korean medical } \\
\text { students: } \\
\text { Findings from a } \\
\text { nationwide } \\
\text { survey [48] }\end{array}$ & Park et al. 2015 & Korea & $\begin{array}{l}\text { Investigate } \\
\text { empathy scores } \\
\text { of medical } \\
\text { students } \\
\text { throughout the } \\
\text { country }\end{array}$ & $\begin{array}{l}\text { Cross- } \\
\text { sectional }\end{array}$ & $5521(39 \%)$ & JSPE-S & $\begin{array}{l}\text { - Lower } \\
\text { empathy score } \\
\text { in males, those } \\
\text { in higher } \\
\text { grades, and } \\
\text { among } \\
\text { undergraduate } \\
\text { students }\end{array}$ & $\begin{array}{l}\text { - Multi-center } \\
\text { survey - Low } \\
\text { response } \\
\text { rate }\end{array}$ \\
\hline $\begin{array}{l}\text { Empathy in } \\
\text { senior year and } \\
\text { first year medical } \\
\text { students: a }\end{array}$ & $\begin{array}{l}\text { Magalhães et al. } \\
2011\end{array}$ & Portugal & $\begin{array}{l}\text { Investigate the } \\
\text { differences in } \\
\text { empathy scores } \\
\text { between first- }\end{array}$ & $\begin{array}{l}\text { Cross- } \\
\text { sectional }\end{array}$ & 476 (92\%) & JSPE-S & $\begin{array}{l}\text { - Sixth-year stu- } \\
\text { dents had } \\
\text { higher em- } \\
\text { pathy scores }\end{array}$ & $\begin{array}{l}\text { - Single- } \\
\text { institution } \\
\text { survey }\end{array}$ \\
\hline
\end{tabular}


Table 1 Results (Continued)

\begin{tabular}{|c|c|c|c|c|c|c|c|c|}
\hline Title & $\begin{array}{l}\text { Author, year } \\
\text { published }\end{array}$ & Country & Study objective & $\begin{array}{l}\text { Study } \\
\text { design }\end{array}$ & $\begin{array}{l}\text { Sample size } \\
\text { (response rate) }\end{array}$ & Scales $^{1}$ & Results & $\begin{array}{l}\text { Comments on } \\
\text { study quality }\end{array}$ \\
\hline $\begin{array}{l}\text { cross-sectional } \\
\text { study [27] }\end{array}$ & & & $\begin{array}{l}\text { year and senior } \\
\text { students, be- } \\
\text { tween genders, } \\
\text { and between } \\
\text { specialty } \\
\text { preferences }\end{array}$ & & & & $\begin{array}{l}\text { than first-year } \\
\text { medical stu- } \\
\text { dents - Fe- } \\
\text { males had } \\
\text { higher em- } \\
\text { pathy scores } \\
\text { than males - } \\
\text { No significant } \\
\text { difference in } \\
\text { empathy } \\
\text { scores among } \\
\text { different speci- } \\
\text { ality } \\
\text { preferences }\end{array}$ & \\
\hline $\begin{array}{l}\text { Empathy in UK } \\
\text { medical } \\
\text { students: } \\
\text { differences by } \\
\text { gender, medical } \\
\text { year and } \\
\text { specialty interest } \\
\text { [49] }\end{array}$ & Tavakol et al. 2011 & $\begin{array}{l}\text { United } \\
\text { Kingdom }\end{array}$ & $\begin{array}{l}\text { Investigate the } \\
\text { relationship } \\
\text { between } \\
\text { undergraduate } \\
\text { medical } \\
\text { students' } \\
\text { empathy scores }\end{array}$ & $\begin{array}{l}\text { Cross- } \\
\text { sectional }\end{array}$ & $853(68.2 \%)$ & JSPE-S & $\begin{array}{l}\text { - There was no } \\
\text { significant } \\
\text { difference } \\
\text { between the } \\
\text { mean empathy } \\
\text { scores across } \\
\text { year groups - } \\
\text { Females scored } \\
\text { significantly } \\
\text { higher } \\
\text { empathy } \\
\text { scores on the } \\
\text { JSPE-S - Med- } \\
\text { ical students } \\
\text { choosing } \\
\text { people- } \\
\text { orientated spe- } \\
\text { cialties were } \\
\text { more empathic } \\
\text { than students } \\
\text { choosing } \\
\text { technology- } \\
\text { orientated } \\
\text { specialties }\end{array}$ & $\begin{array}{l}\text { - Single- } \\
\text { institution } \\
\text { survey - } \\
\text { Missing data } \\
\text { were re- } \\
\text { placed with } \\
\text { the mean of } \\
\text { all values }\end{array}$ \\
\hline $\begin{array}{l}\text { Empathy score } \\
\text { among medical } \\
\text { students in } \\
\text { Mashhad, Iran: } \\
\text { study of the } \\
\text { Jefferson Scale } \\
\text { of Physician [50] }\end{array}$ & Rezayat et al. 2018 & Iran & $\begin{array}{l}\text { Investigate } \\
\text { psychometric } \\
\text { characteristics of } \\
\text { the JSPE scale } \\
\text { among medical } \\
\text { students in } \\
\text { Mashhad, Iran }\end{array}$ & $\begin{array}{l}\text { Cross- } \\
\text { sectional }\end{array}$ & 640 & JSPE-S & $\begin{array}{l}\text { - Empathy score } \\
\text { among } \\
\text { medical } \\
\text { students } \\
\text { decreased } \\
\text { when their } \\
\text { educational } \\
\text { years increased } \\
\text { - Higher } \\
\text { empathy } \\
\text { scores in } \\
\text { females than in } \\
\text { male students } \\
\text { - The overall } \\
\text { rate of } \\
\text { empathy in } \\
\text { basic sciences } \\
\text { period was } \\
\text { more than in } \\
\text { the clinical } \\
\text { period - } \\
\text { Empathy } \\
\text { decreased with } \\
\text { increasing age }\end{array}$ & $\begin{array}{l}\text { - Single- } \\
\text { Institution } \\
\text { survey. - No } \\
\text { response } \\
\text { rate - Selec- } \\
\text { tion bias due } \\
\text { to exclusion } \\
\text { criteria }\end{array}$ \\
\hline $\begin{array}{l}\text { Erosion of } \\
\text { empathy during } \\
\text { medical training } \\
\text { by gender. A }\end{array}$ & $\begin{array}{l}\text { Calzadilla-Núñez } \\
\text { et al. } 2017\end{array}$ & $\begin{array}{l}\text { Colombia } \\
\text { Ecuador }\end{array}$ & $\begin{array}{l}\text { Investigate } \\
\text { whether } \\
\text { empathic } \\
\text { erosion is a }\end{array}$ & $\begin{array}{l}\text { Cross- } \\
\text { sectional }\end{array}$ & $\begin{array}{l}\text { University 1: } 278 \\
(98 \%) \text { University } \\
\text { 2: } 756 \text { (77.86\%) }\end{array}$ & JSPE-S & $\begin{array}{l}\text { - There are no } \\
\text { general } \\
\text { patterns of } \\
\text { how overall }\end{array}$ & $\begin{array}{l}\text { - Multi-center } \\
\text { survey - } \\
\text { Some educa- } \\
\text { tional years }\end{array}$ \\
\hline
\end{tabular}


Table 1 Results (Continued)

\begin{tabular}{|c|c|c|c|c|c|c|c|c|}
\hline Title & $\begin{array}{l}\text { Author, year } \\
\text { published }\end{array}$ & Country & Study objective & $\begin{array}{l}\text { Study } \\
\text { design }\end{array}$ & $\begin{array}{l}\text { Sample size } \\
\text { (response rate) }\end{array}$ & Scales ${ }^{1}$ & Results & $\begin{array}{l}\text { Comments on } \\
\text { study quality }\end{array}$ \\
\hline $\begin{array}{l}\text { cross-sectional } \\
\text { study [51] }\end{array}$ & & & $\begin{array}{l}\text { general } \\
\text { phenomenon in } \\
\text { the schools of } \\
\text { medicine } \\
\text { included in the } \\
\text { study and its } \\
\text { relation to } \\
\text { gender }\end{array}$ & & & & $\begin{array}{l}\text { empathy and } \\
\text { its } \\
\text { components } \\
\text { behave over } \\
\text { school years - } \\
\text { Males and } \\
\text { females do not } \\
\text { have the same } \\
\text { empathic } \\
\text { response }\end{array}$ & $\begin{array}{l}\text { have small } \\
\text { sample sizes } \\
\text { - No assess- } \\
\text { ment of stat- } \\
\text { istical } \\
\text { significance }\end{array}$ \\
\hline $\begin{array}{l}\text { How well do } \\
\text { medical students } \\
\text { rate and } \\
\text { communicate } \\
\text { clinical } \\
\text { empathy? [52] }\end{array}$ & Lim et al. 2013 & $\begin{array}{l}\text { New } \\
\text { Zealand }\end{array}$ & $\begin{array}{l}\text { Investigate the } \\
\text { teaching, } \\
\text { learning and } \\
\text { communication } \\
\text { skills of clinical } \\
\text { empathy in New } \\
\text { Zealand medical } \\
\text { students; in } \\
\text { particular to } \\
\text { determine if } \\
\text { there is a similar } \\
\text { decline during } \\
\text { training in that } \\
\text { country, and } \\
\text { how well } \\
\text { students, peers, } \\
\text { and tutors } \\
\text { recognise good } \\
\text { skills and } \\
\text { communication } \\
\text { of empathy }\end{array}$ & Longitudinal & 72 & $\begin{array}{l}\text { JSPE-S= } \\
\text { BECCI }\end{array}$ & $\begin{array}{l}\text { - Self-reported } \\
\text { empathy de- } \\
\text { clines during } \\
\text { undergraduate } \\
\text { medical train- } \\
\text { ing - No signifi- } \\
\text { cant gender } \\
\text { difference in } \\
\text { self-reported } \\
\text { empathy } \\
\text { scores was } \\
\text { observed }\end{array}$ & $\begin{array}{l}\text { - Single- } \\
\text { institution } \\
\text { survey }\end{array}$ \\
\hline $\begin{array}{l}\text { Level of } \\
\text { Empathy among } \\
\text { Medical Students } \\
\text { in Kuwait } \\
\text { University [53] }\end{array}$ & Hasan et al. 2013 & Kuwait & $\begin{array}{l}\text { Investigate the } \\
\text { level of } \\
\text { empathy among } \\
\text { medical } \\
\text { students at } \\
\text { various years of } \\
\text { study and other } \\
\text { factors }\end{array}$ & $\begin{array}{l}\text { Cross- } \\
\text { sectional }\end{array}$ & $264(56 \%)$ & $\begin{array}{l}\text { JSPE- } \\
\text { SZKPQ-50- } \\
\text { CCPSS-10 }\end{array}$ & $\begin{array}{l}\text { - Increasing } \\
\text { empathy level } \\
\text { with the } \\
\text { academic year, } \\
\text { which peaked } \\
\text { in the 4th-year, } \\
\text { followed by a } \\
\text { slight drop in } \\
\text { subsequent } \\
\text { years - A } \\
\text { higher level of } \\
\text { empathy } \\
\text { among females } \\
\text { - The desired } \\
\text { specialty was } \\
\text { not signifi- } \\
\text { cantly associ- } \\
\text { ated with } \\
\text { levels of } \\
\text { empathy }\end{array}$ & $\begin{array}{l}\text { - Single- } \\
\text { institution } \\
\text { survey - A } \\
\text { non- } \\
\text { validated } \\
\text { Arabic ver- } \\
\text { sion of em- } \\
\text { pathy scales }\end{array}$ \\
\hline $\begin{array}{l}\text { Maintaining } \\
\text { empathy in } \\
\text { medical school: } \\
\text { It is possible [24] }\end{array}$ & Hegazi et al. 2013 & Australia & $\begin{array}{l}\text { Investigate } \\
\text { levels of } \\
\text { empathy in } \\
\text { University of } \\
\text { Western Sydney } \\
\text { (UWS) Medical } \\
\text { School students } \\
\text { across the } \\
\text { different years } \\
\text { of } \\
\text { undergraduate } \\
\text { medical } \\
\text { education }\end{array}$ & $\begin{array}{l}\text { Cross- } \\
\text { sectional }\end{array}$ & 404 (69.78\%) & JSPE-S & $\begin{array}{l}\text { - No significant } \\
\text { difference in } \\
\text { empathy } \\
\text { scores in } \\
\text { relation to the } \\
\text { year of the } \\
\text { medical course } \\
\text { - Gender } \\
\text { difference in } \\
\text { levels of } \\
\text { empathy, } \\
\text { favouring } \\
\text { females }\end{array}$ & $\begin{array}{l}\text { - Single- } \\
\text { institution } \\
\text { survey. }\end{array}$ \\
\hline Malaysian & Williams et al. & Malaysia & Investigate & Cross- & 1st year: 122 & JSPE-S & - Self-reported & - Single- \\
\hline
\end{tabular}


Table 1 Results (Continued)

\begin{tabular}{|c|c|c|c|c|c|c|c|c|}
\hline Title & $\begin{array}{l}\text { Author, year } \\
\text { published }\end{array}$ & Country & Study objective & $\begin{array}{l}\text { Study } \\
\text { design }\end{array}$ & $\begin{array}{l}\text { Sample size } \\
\text { (response rate) }\end{array}$ & Scales $^{1}$ & Results & $\begin{array}{l}\text { Comments on } \\
\text { study quality }\end{array}$ \\
\hline $\begin{array}{l}\text { Medical } \\
\text { Students' self- } \\
\text { reported Em- } \\
\text { pathy: A cross- } \\
\text { sectional Com- } \\
\text { parative Study } \\
\text { [54] }\end{array}$ & 2015 & & $\begin{array}{l}\text { empathy levels } \\
\text { between first- } \\
\text { year and } \\
\text { second-year } \\
\text { medical } \\
\text { students }\end{array}$ & sectional & $\begin{array}{l}\text { (100\%) 2nd year: } \\
71(70 \%)\end{array}$ & & $\begin{array}{l}\text { empathy levels } \\
\text { declined sig- } \\
\text { nificantly from } \\
\text { first-year to } \\
\text { second-year - } \\
\text { No significant } \\
\text { differences in } \\
\text { empathy } \\
\text { scores be- } \\
\text { tween genders }\end{array}$ & $\begin{array}{l}\text { institution } \\
\text { survey }\end{array}$ \\
\hline $\begin{array}{l}\text { Rethinking } \\
\text { empathy } \\
\text { decline: results } \\
\text { from an OSCE } \\
{[28]}\end{array}$ & Teng et al. 2017 & USA & $\begin{array}{l}\text { Investigate } \\
\text { observed } \\
\text { empathy among } \\
\text { medical } \\
\text { students in } \\
\text { different } \\
\text { clerkship years } \\
\text { using an OSCE }\end{array}$ & $\begin{array}{l}\text { Cross- } \\
\text { sectional }\end{array}$ & 129 & MPCC & $\begin{array}{l}\text { - Found a } \\
\text { possible trend } \\
\text { towards higher } \\
\text { MPCC among } \\
\text { students in } \\
\text { their second } \\
\text { clerkship year } \\
\text { compared with } \\
\text { students in } \\
\text { their first-year } \\
\text { ( } p=0.09 \text { ), } \\
\text { which became } \\
\text { more signifi- } \\
\text { cant when } \\
\text { adjusting for } \\
\text { outliers ( } p= \\
\text { 0.05) - There } \\
\text { was no differ- } \\
\text { ence in per- } \\
\text { formance by } \\
\text { gender. - Stu- } \\
\text { dents who } \\
\text { intended to } \\
\text { pursue a } \\
\text { "people-orien- } \\
\text { tated" specialty } \\
\text { score higher in } \\
\text { handling the } \\
\text { patient's } \\
\text { frustration }\end{array}$ & $\begin{array}{l}\text { - Single- } \\
\text { institution } \\
\text { survey - } \\
\text { Small sample } \\
\text { size }\end{array}$ \\
\hline $\begin{array}{l}\text { Stability of } \\
\text { empathy among } \\
\text { undergraduate } \\
\text { medical } \\
\text { students: A } \\
\text { longitudinal } \\
\text { study at one UK } \\
\text { medical school } \\
\text { [55] }\end{array}$ & Quince et al. 2011 & $\begin{array}{l}\text { United } \\
\text { Kingdom }\end{array}$ & $\begin{array}{l}\text { Investigate the } \\
\text { following } \\
\text { questions: } \\
\text { 1. Do men and } \\
\text { women medical } \\
\text { students differ } \\
\text { in empathy? } \\
\text { 2. Does } \\
\text { empathy } \\
\text { change } \\
\text { amongst men } \\
\text { and women } \\
\text { over time? }\end{array}$ & Longitudinal & 1653 & $|\mathrm{R}|$ & $\begin{array}{l}\text { - Compared to } \\
\text { females, males } \\
\text { recorded lower } \\
\text { levels of } \\
\text { affective } \\
\text { empathy } \\
\text { throughout } \\
\text { their course } \\
\text { and lower } \\
\text { levels of } \\
\text { cognitive } \\
\text { empathy for } \\
\text { part of their } \\
\text { medical course } \\
\text { - Male's } \\
\text { affective } \\
\text { empathy } \\
\text { declined } \\
\text { slightly across } \\
\text { the course } \\
\text { overall, while } \\
\text { female's } \\
\text { affective } \\
\text { empathy } \\
\text { showed no } \\
\text { change. }\end{array}$ & $\begin{array}{l}\text { - Single- } \\
\text { institution } \\
\text { survey }\end{array}$ \\
\hline
\end{tabular}


Table 1 Results (Continued)

\begin{tabular}{|c|c|c|c|c|c|c|c|c|}
\hline Title & $\begin{array}{l}\text { Author, year } \\
\text { published }\end{array}$ & Country & Study objective & $\begin{array}{l}\text { Study } \\
\text { design }\end{array}$ & $\begin{array}{l}\text { Sample size } \\
\text { (response rate) }\end{array}$ & Scales $^{1}$ & Results & $\begin{array}{l}\text { Comments on } \\
\text { study quality }\end{array}$ \\
\hline & & & & & & & $\begin{array}{l}\text { Neither male } \\
\text { nor female } \\
\text { showed any } \\
\text { change in } \\
\text { cognitive } \\
\text { empathy } \\
\text { during the } \\
\text { course }\end{array}$ & \\
\hline $\begin{array}{l}\text { The complexity } \\
\text { of empathy } \\
\text { during medical } \\
\text { school training: } \\
\text { Evidence for } \\
\text { positive changes } \\
\text { [56] }\end{array}$ & Smith et al. 2017 & USA & $\begin{array}{l}\text { Investigate } \\
\text { multiple facets } \\
\text { of empathy }\end{array}$ & Longitudinal & 122 & $\begin{array}{l}\text { JSPE- } \\
\text { SQCAE }\end{array}$ & $\begin{array}{l}\text { - JSPE-S em- } \\
\text { pathy scores } \\
\text { decreased } \\
\text { throughout } \\
\text { training - Stu- } \\
\text { dents exhibited } \\
\text { an increase in } \\
\text { QCAE total } \\
\text { score over time } \\
\text { - Females ex- } \\
\text { hibited higher } \\
\text { levels of self- } \\
\text { reported } \\
\text { empathy }\end{array}$ & $\begin{array}{l}\text { - Single- } \\
\text { institution } \\
\text { survey - } \\
\text { Small } \\
\text { sample-size }\end{array}$ \\
\hline
\end{tabular}

JSPE-S: Jefferson Scale of Empathy - Student Version: Measures affective empathy. The scale studies the empathic relationship between the medical student and the patient. It consists of 20 items on a 7-point Likert scale, with a minimum of 20 and a maximum of 140 points. A high score indicates a more empathic orientation [57]

NEO-Five Factor Inventory: Measures personality. It consists of 60 items that depict the following five dimensions: Neuroticism, Extraversion, Agreeableness, Openness to Experience and Consciousness. Responders answer the degree of agreement or disagreement on a 5-point Likert scale from 0 to 4 [58] TEQ: Toronto Empathy Questionnaire: A self-reported scale. Consists of 16 questions which are designed to measure the affective component of empathy. The questions are scored on a 5-point Likert scale with a total score out of 64. A high total score accounts for a high level of emotional empathy [34] RMET: Reading the mind in the eyes: Measures cognitive empathy by depicting the eye region in 36 photographs. The participant is required to identify the emotion being expressed and select an answer from four options. One point for each correct answer. The maximum score is 36 with mean scores typically ranging from 24 to 30 [34]

BEES: Balanced Emotional Empathy Scale: Measures affective empathy by evaluating responses to different fictive situations and life events. It consists of 30 items of which 15 are positively worded and 15 are negatively worded. Responders answer the degree of agreement or disagreement on a 9-point Likert-scale. A high total score accounts for a high level of emotional empathy [37]

OSCE: Objective structured clinical examinations [38]

Empathic Skill Scale B Form: Measures empathic skill level. It consists of six psychological problems concerning daily life and 12 types of reactions to each of these problems. Responders answer on a Likert type scale. The highest achievable score is 219 and the lowest score is 64 [23]

Conflict Tendency Scale: Measures adults' communication skills and characterise their conflicts. It consists of 53 items - 31 positives and 22 negatives. The positive items depict a communication conflict. Negative items depict no conflict [23]

IRI: Interpersonal Reactivity Index: Consists of four scales which includes seven items - in total 28 items. The four scales consist of 'Perspective taking'; 'Empathic concern'; 'Fantasy' and 'Personal distress'. Altogether these items embrace some of the cornerstones of empathy [59, 60]

BECCl: Behaviour Change Counselling Index: Measure of practitioner's consultation skills about behaviour change. It consists of 11 items. An example of an item is: "Practitioner demonstrates sensitivity to talking about other issues". Responders answer on a 5-point-Likert scale ranging from 0 to 4 [52]

ZKPQ-50-CC: Zuckermann-Kuhlman Personality Questionnaire: Measures personality types. It consists of 50 true-or-false questions that depict the following five dimensions: aggression and hostility, impulsive sensation seeking, neuroticism and anxiety, activity and sociability [61]

PSS-10: Perceived Stress Scale 10-item version: Measures the level of stress. It consists of 10 items that depict the level of stress and the handling of this. A high score indicates a high level of stress [62]

MPCC: Measure of Patient Centered Communication: Measures observed empathy. It consists of three dimensions: 1) Exploring the disease and illness experience; 2) Understanding the whole person, and; 3) Finding common ground [63]

QCAE: Questionnaire of Cognitive and Affective empathy: Measures affective and cognitive components of empathy. It consists of 31 items that depict five subscales: Perspective taking, Online Stimulation, Emotion Contagion, Proximal Responsivity, Peripheral Responsivity. Responders answer their degree of agreement or disagreement on a 4-point Likert scale [64]

empathy scores among second-year students compared to third-year students and the opposite for the observed empathy scores. In another longitudinal study by Chen et al. [35] higher levels of empathy were found up to the thirdyear of education, followed by a persistent decline.

Smith et al. [56] conducted a longitudinal study applying both JSPE-S and the Questionnaire of Cognitive and Affective Empathy (QCAE). The two scales revealed incongruent results: the QCAE score increased over time while JSPE-S measured a decrease over time.

\section{Gender}

Female students were reported to have higher empathy scores compared to male students in 16 cross-sectional and 2 longitudinal studies [21, 24, 27, 34-37, 39, 40, 42$44,47-50,53,56]$. One longitudinal study by Quince et al. [55] found a lower level of emotional empathy among men compared to women who did not show any change. No gender differences were found in relation to cognitive empathy and no differences between genders were detected in seven cross-sectional [23, 28, 33, 41, 45, 
46, 54]. Three studies did not investigate the differences in empathy across genders $[32,38,51]$.

\section{Specialty preferences}

Nine cross-sectional studies investigated a possible relation between empathy scores and specialty preferences of the students [27, 28, 33, 34, 39, 43, 45, 46, 53]. Three studies detected higher levels of empathy among students who preferred a "people-orientated" specialty [28, 43, 45]. No association between specialty preferences and empathy scores was found in the remaining six studies. None of the longitudinal studies examined specialty preferences.

\section{Western and non-Western countries}

Out of the thirty studies, nine cross-sectional studies that all applied JSPE-S, from India [36, 39], Kuwait [53], China [44, 45], Korea [48], Iran [46, 50] and Pakistan [33], reported lower mean empathy scores compared to Western countries.

\section{Discussion}

\section{Main findings}

This systematic review aimed to investigate the level of empathy among medical students across the educational years and how the measured empathy levels relate to gender, specialty preferences, and nationality. In reviewing studies from 20 different countries, variations were found in the level of empathy among medical students across the number of educational years. Nearly half of the included studies $[21,33,34,39-41,45-48,50,52$, $54,56]$, of which only two $[52,56]$ were longitudinal, reported lower empathy scores with higher educational years. The remaining 17 studies [23, 24, 27, 28, 32, 35$38,42-44,49,51,53,55,56]$ identified both higher, mixed or unchanged levels of empathy throughout the medical education.

Most studies [21, 24, 27, 34-37, 39, 40, 42-44, 47-50, $53,55,56]$ found a tendency towards higher levels of empathy among female students as compared to male students. Out of nine cross-sectional studies, only three [28, 43, 45] reported an association between empathy and specialty preferences. Furthermore, studies from non-Western countries reported a lower level of mean empathy scores as compared to Western countries. These findings thus differed from the previous review by Neumann et al. [25] which concluded that empathy decreases by an increase in the educational years particularly among those preferring "non-people-orientated" specialities. While different results might be explained by differences in study populations, study design (longitudinal vs cross-section), the instrument used, local culture, etc., this review tells us that we cannot make the often quoted statement that "empathy declines with level of training".

\section{Possible explanations for lower and higher levels of empathy}

In the literature, several explanations for a decline in empathy have been discussed without demonstrating a clear causal relationship. Some scholars point to the phenomenon of burnout among medical students and refer to the association found in the literature between high burnout level among medical students and low empathy score [65-67]. Related, stress among medical students [68-70] has also been shown to correlate negatively with empathy [69]. Another explanation put forward in the literature for empathy decline is increased patient contact during clinical training [34, 35, $38,45,46,50,52]$. Chen et al. [38] explained the development towards lower levels of empathy during clinical training as a result of an acculturation process in which superiors and mentors try to protect their students against psychological distress by cultivating a climate of cynicism, emotional distance and detachment among medical students in their contact with patients and at the same time try to safeguard "professionalism" in the clinical setting. Moreover, Li et al. [45] stated that clinical training might encompass intense patient-physician relationships, long working hours and sleep deprivation, leading to lower levels of empathy after clinical training. Furthermore, in the literature, the so-called "hidden curriculum," lack of role models, fear and anxiety in the meeting with the patients, and increased workload are also pointed out as possible reasons for a decline in empathy [46, 71, 72]. Another explanation mentioned in the literature is that the medical curriculum focuses more on diagnosis and treatment than humanistic values [73]. Shapiro et al. [71] also stated that the biomedical discourse has diverted the students' focus from empathy leading him/her to adopt a mechanistic view on illness that might reduce the patients to a disease or an object.

Discussing the increases in empathy levels that were documented in some of the reviewed studies, Magalhaes et al. [27] pointed out that the medical curriculum has an increased focus on the development of empathy as the educational years progress and that students have increasingly reached an acknowledgment of the importance of empathy in the patientphysician relationship. This point of view was put forward as a possible explanation for the documented increases in empathy. Furthermore, training and competence acquirement through clinical training of communication skills have also been proposed as an explanation for the tendency towards higher levels of empathy in senior year medical students [27, 28]. In relation to these explanations it should also be kept in mind that the medical curriculum varies across countries and medical schools. 


\section{Gender differences}

In the literature, varying explanations for gender differences are suggested. Bertakis et al. [16] found that females are more receptive to emotional signals than males. Furthermore, they are said to show more interest in the patient's family and social life, thereby being able to achieve a better understanding of the patient and reach a more empathic relation. Shashikumar et al. [39] stated that females through evolutionary gender differences are more caring and loving.

\section{Nationalities}

Nine studies in our systematic review reported a propensity towards lower empathy scores in non-Western compared to Western countries. All of these studies applied the JSPE-S. Shariat et al. [47] stated that awareness of the cultural differences should be kept in mind when applying the JSPE-S in cultures that differ from the USA, where the JSPE-S was developed. A Japanese psychometric study of the JSPE pointed out that Japanese patients preferred their physician to be calm and unemotional, emphasising that cultural differences could indeed explain the differences in empathy scores between countries and cultures [74].

\section{Specialty preferences}

A possible association between levels of empathy and specialty preferences was investigated in nine of the included studies [27, 28, 33, 34, 39, 43, 45, 46, 53]. Only three studies $[28,43,45]$ reported an association between higher level of empathy among people preferring "people-orientated" specialities. Engaging in an empathic understanding of the patients' feelings and life circumstances is important in all medical specialities since showing an empathic attitude towards the patient has been shown to lead to positive effects on patients' health outcome $[8,9]$. It can be argued, however, that a focus on empathy is relevant mostly within people-orientated specialities since physicians who work in these specialities are both in need of help regarding empathy preservation (helping patients) and administration (helping themselves so as to decrease the risk of stress and burnout) [3].

\section{Strengths and limitations}

A strength of the present systematic review is that the literature search was conducted in three databases. Furthermore, the screening of literature and selection of studies was performed by two reviewers. Moreover, we consider the implementation of a quality assessment of all included studies as a strength. This review has several limitations. Since our search words included words that presume a change e.g. "decrease" and "increase," our search may be too narrow, and there is a risk that relevant studies have been overlooked. Additionally, possible relevant studies in languages other than English and Scandinavian were not included. Another limitation is that only quantitative studies were included. This excluded qualitative aspects that could have contributed to a more varied and profound understanding of the quantitative findings.

\section{Future research}

Most of the included studies applied the selfadministered JSPE-S and therefore did not explore the display of empathy that takes place between the patient and the medical student. Sulzer et al. [75] stated that the JSPE-S scale focuses on thoughts and not actions. Furthermore, research has shown that self-reported empathy has only a vague association with the patientphysician relationship in the clinical setting [75]. To improve the knowledge of empathy among medical students, research that includes both cognition, action, and feelings is recommended [75]. Incorporation of non-medical students as control groups is also required in order to gain more insight into whether medical students' levels of empathy differ compared to other university students. Furthermore, future investigators should employ a variety of research designs to investigate the important role of empathy in medical education, such as mixed methods research, observational research, and qualitative research. These studies could focus - not on self-reporting - but rather on patient perceptions of empathic student/physician behaviour. Qualitative research conducted with students could also contribute to new perspectives and insights about student-perceived factors influencing the development of empathy and its expression in clinical care. Lastly, a meta-analysis is desirable since it enables the calculation of statistical significance and heterogeneity.

\section{Conclusions}

This systematic review including thirty studies, revealed varied and inconsistent findings on the levels of empathy among medical students. Statistically lower empathy scores by an increase in educational years were found in 14 studies. The remaining studies reported higher [4] and unchanged [6] scores in empathy. In most studies, females were reported to have higher levels of empathy than males. Study participants from non-Western countries reported a tendency towards lower mean empathy scores as compared to those from Western countries. Only a few studies reported a correlation between "people-oriented" specialty preferences and empathy scores. Future research should focus on examining relational empathy in studentpatient interaction using observational scales and qualitative methodologies. 


\section{Supplementary information}

Supplementary information accompanies this paper at https://doi.org/10. 1186/s12909-020-1964-5.

Additional file 1. Search protocol. Overview of the search strategy employed in the following databases PubMed, Embase and PsycINFO.

Additional file 2. Methodological quality assessment of the included studies. Above each table the type of quality assessment tool is indicated. In the first table (with overview of the longitudinal studies) the criterions appear in the left column and for each included study there is answered "yes" or "no" to these criterions. In the second table (with overview of the cross-sectional studies) the studies appear in the left column and the criteria items in the first row of the table. The quality assessment tool here is indicated with $0,0.5$ or 1 point per item. The total score is indicated in the right column.

\section{Abbreviations}

BEES: Balanced Emotional Empathy Scale; CARE: Consultation-and-RelationalEmpathy; IRI: Interpersonal Reactivity Index scale; JSPE: Jefferson Scale of Physician Empathy; JSPE-S: Jefferson Scale of Physician Empathy student version; LDL: Lipoprotein cholesterol; MeSH: Medical Subject Heading terms; MPCC: Measure of Patient-Centered Communication; OSCE: Objective Structured Clinical Examinations; PRISMA: Preferred Reporting Items for Systematic Reviews and Meta-Analyses; QCAE: Questionnaire of Cognitive and Affective Empathy; RMET: Reading the Mind in the Eyes

\section{Authors' contributions}

AJ and FA participated in the design of the study, developed the search strategy, extracted the data and drafted the manuscript. CMA and EAH participated in the design of the study and helped to draft the manuscript. All authors (AJ, FA, JS, EAH, CMA) declare that they have read and approved the final manuscript. AJ and FA: graduate medical students. JS, CMA and EAH: senior researchers.

\section{Funding}

Not applicable.

\section{Availability of data and materials}

All data generated or analysed during this study are included in this published article.

\section{Ethics approval and consent to participate} Not applicable.

\section{Consent for publication}

Not applicable.

\section{Competing interests}

The authors declare that they have no competing interests.

\section{Author details}

'University of Southern Denmark, Campusvej 55, 5230 Odense M, Denmark. ${ }^{2}$ Research Unit of General Practice, Department of Public Health, University of Southern Denmark, J.B. Winsløws Vej 9A, 5000 Odense C, Denmark. ${ }^{3}$ Department of Psychology, University of Southern Denmark, Campusvej 55, 5230 Odense M, Denmark. ${ }^{4}$ Department for the Study of Culture, University of Southern Denmark, Campusvej 55, 5230 Odense M, Denmark.

\section{Received: 30 August 2019 Accepted: 7 February 2020} Published online: 17 February 2020

\section{References}

1. Mackay R, Hughes JR, Carver EJ. Empathy in the helping relationship. New York: Springer; 1990

2. Mercer SW, Reynolds WJ. Empathy and quality of care. Br J Gen Pract. 2002; 52(Suppl):S9-12.

3. Hojat M. Empathy in health professions education and patient care Swizterland: Springer; 2007.
4. Sadati AK, Tabei SZ, Lankarani KB. A qualitative study on the importance and value of doctor-patient relationship in Iran: physicians' views. Open Access Maced J Med Sci. 2018;6(10):1895-901.

5. Derksen F, Bensing J, Kuiper S, van Meerendonk M, Lagro-Janssen A. Empathy: what does it mean for GPs? A qualitative study. Fam Pract. 2015; 32(1):94-100.

6. Derksen F, Olde Hartman TC, van Dijk A, Plouvier A, Bensing J, LagroJanssen A. Consequences of the presence and absence of empathy during consultations in primary care: a focus group study with patients. Patient Educ Couns. 2017;100(5):987-93.

7. Squier RW. A model of empathic understanding and adherence to treatment regimens in practitioner-patient relationships. Soc Sci Med. 1990; 30(3):325-39.

8. Steinhausen S, Ommen O, Thum S, Lefering R, Koehler T, Neugebauer E, et al. Physician empathy and subjective evaluation of medical treatment outcome in trauma surgery patients. Patient Educ Couns. 2014;95(1):53-60.

9. Hojat M, Louis DZ, Markham FW, Wender R, Rabinowitz C, Gonnella JS. Physicians' empathy and clinical outcomes for diabetic patients. Acad Med. 2011;86(3):359-64

10. Renzi C, Abeni D, Picardi A, Agostini E, Melchi CF, Pasquini P, et al. Factors associated with patient satisfaction with care among dermatological outpatients. Br J Dermatol. 2001;145(4):617-23.

11. Kim SS, Kaplowitz S, Johnston MV. The effects of physician empathy on patient satisfaction and compliance. Eval Health Prof. 2004;27(3):237-51.

12. Mercer SW, Watt GC, Reilly D. Empathy is important for enablement. BMJ. 2001:322(7290):865.

13. Larson EB, Yao X. Clinical empathy as emotional labor in the patientphysician relationship. JAMA. 2005;293(9):1100-6.

14. Thirioux B, Birault F, Jaafari N. Empathy is a protective factor of burnout in physicians: new neuro-phenomenological hypotheses regarding empathy and sympathy in care relationship. Front Psychol. 2016;7:763.

15. Hojat M, Zuckerman M, Gonnella JS, Mangione S, Nasca TJ, Vergare MJ, et al. Empathy in medical students as related to specialty interest personality, and perception of mother and father. Personal Individ Differ. 2005;39:1205-15.

16. Bertakis KD, Helms LJ, Callahan EJ, Azari R, Robbins JA. The influence of gender on physician practice style. Med Care. 1995;33(4):407-16.

17. Roter DL, Hall JA. Physician gender and patient-centered communication: a critical review of empirical research. Annu Rev Public Health. 2004;25:497-519.

18. Shanafelt TD, West C, Zhao X, Novotny P, Kolars J, Habermann T, et al. Relationship between increased personal well-being and enhanced empathy among internal medicine residents. J Gen Intern Med. 2005;20(7):559-64.

19. Ferreira-Valente A, Monteiro JS, Barbosa RM, Salgueira A, Costa P, Costa MJ. Clarifying changes in student empathy throughout medical school: a scoping review. Adv Health Sci Educ Theory Pract. 2017;22(5):1293-313.

20. Chen D, Lew R, Hershman W, Orlander J. A cross-sectional measurement of medical student empathy. J Gen Intern Med. 2007:22(10):1434-8.

21. Khademalhosseini M, Khademalhosseini Z, Mahmoodian F. Comparison of empathy score among medical students in both basic and clinical levels. J Adv Med Educ Prof. 2014;2(2):88-91.

22. Todres M, Tsimtsiou Z, Stephenson A, Jones $R$. The emotional intelligence of medical students: an exploratory cross-sectional study. Med Teach. 2010; 32(1):e42-8.

23. Atay IM, Sari M, Demirhan M, Aktepe E. Comparison of empathy skills and conflict tendency in preclinical and clinical phase Turkish medical students: a cross-sectional study. Dusunen Adam. 2014;27(4):308-15.

24. Hegazi I, Wilson I. Maintaining empathy in medical school: it is possible. Med Teach. 2013;35(12):1002-8.

25. Neumann M, Edelhauser F, Tauschel D, Fischer MR, Wirtz M, Woopen C, et al. Empathy decline and its reasons: a systematic review of studies with medical students and residents. Acad Med. 2011;86(8):996-1009.

26. Colliver JA, Conlee MJ, Verhulst SJ, Dorsey JK. Reports of the decline of empathy during medical education are greatly exaggerated: a reexamination of the research. Acad Med. 2010:85(4):588-93.

27. Magalhaes E, Salgueira AP - Costa P, Costa MJ. Empathy in senior year and first year medical students: a cross-sectional study. BMC Med Educ 2011 11(52):1472-6920

28. Teng VC, Nguyen C, Hall KT, Rydel T, Sattler A, Schillinger E, et al. Rethinking empathy decline: results from an OSCE. Clin Teach. 2017;14(6):441-5.

29. Liberati A, Altman DG, Tetzlaff J, Mulrow C, Gotzsche PC, loannidis JP, et al. The PRISMA statement for reporting systematic reviews and meta-analyses 
of studies that evaluate health care interventions: explanation and elaboration. PLoS Med. 2009;6(7):e1000100.

30. Zeng X, Zhang Y, Kwong JS, Zhang C, Li S, Sun F, et al. The methodological quality assessment tools for preclinical and clinical studies, systematic review and meta-analysis, and clinical practice guideline: a systematic review. J Evid Based Med. 2015;8(1):2-10.

31. Tooth L, Ware R, Bain C, Purdie DM, Dobson A. Quality of reporting of observational longitudinal research. Am J Epidemiol. 2005;161(3):280-8.

32. Costa P, Magalhaes E, Costa MJ. A latent growth model suggests that empathy of medical students does not decline over time. Adv Health Sci Educ Theory Pract. 2013;18(3):509-22.

33. Tariq N, Rasheed T, Tavakol M. A quantitative study of empathy in Pakistani medical students: a multicentered approach. J Prim Care Community Health. 2017:8(4):294-9.

34. Youssef FF, Nunes P, Sa B, Williams S. An exploration of changes in cognitive and emotional empathy among medical students in the Caribbean. Int J Med Educ. 2014:5:185-92.

35. Chen DC, Kirshenbaum DS, Yan J, Kirshenbaum E, Aseltine RH. Characterizing changes in student empathy throughout medical school. Med Teach. 2012;34(4):305-11.

36. Chatterjee A, Ravikumar R, Singh S, Chauhan PS, Goel M. Clinical empathy in medical students in India measured using the Jefferson scale of empathystudent version. J Educ Eval Health Prof. 2017;14:33.

37. Dehning S, Girma E, Gasperi S, Meyer S, Tesfaye M, Siebeck M. Comparative cross-sectional study of empathy among first year and final year medical students in Jimma University, Ethiopia: steady state of the heart and opening of the eyes. BMC Med Educ. 2012;12:34.

38. Chen DC, Pahilan ME, Orlander JD. Comparing a self-administered measure of empathy with observed behavior among medical students. J Gen Intern Med. 2010;25(3):200-2.

39. Shashikumar R, Chaudhary R, Ryali VS, Bhat PS, Srivastava K, Prakash J, et al. Cross sectional assessment of empathy among undergraduates from a medical college. Med J Armed Forces India. 2014;70(2):179-85.

40. Triffaux JM, Tisseron S, Nasello JA. Decline of empathy among medical students: dehumanization or useful coping process? Encephale. 2019;45(1):3-8.

41. Diaz Narvaez VP, Alonso Palacio LM, Caro SE, Silva MG, Castillo JA, Billbao JL, et al. Empathic orientation among medical students from three universities in Barranquilla, Colombia and one university in the Dominican Republic. Arch Argent Pediatr. 2014;112(1):41-9.

42. Quince TA, Kinnersley P, Hales J, da Silva A, Moriarty H, Thiemann P, et al. Empathy among undergraduate medical students: a multi-centre crosssectional comparison of students beginning and approaching the end of their course. BMC Med Educ. 2016;16(92):016-0603.

43. Santos MA, Grosseman S, Morelli TC, Giuliano IC, Erdmann TR. Empathy differences by gender and specialty preference in medical students: a study in Brazil. Int J Med Educ. 2016;7:149-53.

44. Wen D, Ma X, Li H, Liu Z, Xian B, Liu Y. Empathy in Chinese medical students: psychometric characteristics and differences by gender and year of medical education. BMC Med Educ. 2013;13(130):1472-6920.

45. Li D, Xu H, Kang M, Ma SA-OA-OX. Empathy in Chinese eight-year medical program students: differences by school year, educational stage, and future career preference. BMC Med Educ. 2018;18(1):018-1348.

46. Benabbas R. Empathy in Iranian medical students: a comparison by age, gender, academic performance and specialty preferences. Med J Islam Repub Iran. 2016;30:439.

47. Shariat SV, Habibi M. Empathy in Iranian medical students: measurement model of the Jefferson scale of empathy. Med Teach. 2013;35(1):3.

48. Park $\mathrm{KH}$, Roh $\mathrm{H}$, Suh $\mathrm{DH}$, Hojat M. Empathy in Korean medical students: findings from a nationwide survey. Med Teach. 2015;37(10):943-8.

49. Tavakol S, Dennick R, Tavakol M. Empathy in UK medical students: differences by gender, medical year and specialty interest. Educ Prim Care. 2011;22(5):297-303.

50. Rezayat AA, Shahini N, Asl HT, Jarahi L, Behdani F, Shojaei SRH, et al. Empathy score among medical students in Mashhad, Iran: study of the Jefferson scale of physician empathy. Electron Physician. 2018;10(7):7101-6.

51. Calzadilla-Nunez A, Diaz-Narvaez VP, Davila-Ponton Y, Aguilera-Munoz J, Fortich-Mesa N, Aparicio-Marenco D, et al. Erosion of empathy during medical training by gender. A cross-sectional study. Arch Argent Pediatr. 2017:115(6):556-61.

52. Lim BT, Moriarty H - Huthwaite M, Gray L, Pullon S, Gallagher P. How well do medical students rate and communicate clinical empathy? Med Teach. 2013;35(2):3
53. Hasan S, Al-Sharqawi N, Dashti F, AbdulAziz M, Abdullah A, Shukkur M, Bouhaimed $\mathrm{M}$, et al. Level of empathy among medical students in Kuwait University, Kuwait. Med Princ Pract. 2013;22(4):385-9.

54. Williams B, Sadasivan S, Kadirvelu A. Malaysian medical students' selfreported empathy: a cross-sectional comparative study. Med J Malaysia. 2015;70(2):76-80.

55. Quince TA, Parker RA, Wood DF, Benson JA. Stability of empathy among undergraduate medical students: a longitudinal study at one UK medical school. BMC Med Educ. 2011;11(90):1472-6920.

56. KEA-Ohoo S, Norman GJ, Decety J. The complexity of empathy during medical school training: evidence for positive changes. Med Educ. 2017; 51(11):1146-59.

57. Hojat M, Mangione S, Nasca TJ, Rattner S, Erdmann JB, Gonnella JS, et al. An empirical study of decline in empathy in medical school. Med Educ. 2004; 38(9):934-41.

58. Magalhaes E, Salgueira A, Gonzalez A-J, Costa JJ, Costa MJ, Costa P, et al. Psychometric properties of a short personality inventory in Portuguese context. [references]. Psicologia: Reflexao e Critica Vol 2014;27(4):642-57.

59. Hojat M, Mangione S, Kane GC, Gonnella JS. Relationships between scores of the Jefferson Scale of Physician Empathy (JSPE) and the Interpersonal Reactivity Index (IRI). Med Teach. 2005;27(7):625-8.

60. Davis $\mathrm{MH}$. Measuring individual differences in empathy: evidence for a multidimensional approach. J Pers Soc Psychol. 1983;44(1):113-26.

61. Aluja A, Rossier J, Garcia LF, Angleitner A, Kuhlman M, Zuckerman M. A cross-cultural shortened form of the ZKPQ (ZKPQ-50-cc) adapted to English, French, German, and Spanish languages. [references]. Personal Individ Differ. 2006;41(4):619-28

62. Cohen S. Perceived stress in a probability sample of the United States. Spacapan, Shirlynn [Ed]. 1988.

63. Epstein RM, Franks P, Fiscella K, Shields CG, Meldrum SC, Kravitz RL, et al. Measuring patient-centered communication in patient-physician consultations: theoretical and practical issues. Soc Sci Med. 2005;61(7):1516-28.

64. Reniers RL, Corcoran R, Drake R, Shryane NM, Vollm BA. The QCAE: a questionnaire of cognitive and affective empathy. J Pers Assess. 2011;93(1):84-95.

65. Brazeau CM, Schroeder R, Rovi S, Boyd L. Relationships between medical student burnout, empathy, and professionalism climate. Acad Med. 2010; 85(10 Suppl):S33-6.

66. Thomas MR, Dyrbye LN, Huntington JL, Lawson KL, Novotny PJ, Sloan JA, et al. How do distress and well-being relate to medical student empathy? A multicenter study. J Gen Intern Med. 2007;22(2):177-83.

67. Holmes $\mathrm{CL}$, Miller $\mathrm{H}$, Regehr $\mathrm{G}$. (Almost) forgetting to care: an unanticipated source of empathy loss in clerkship. Med Educ. 2017;51(7):732-9.

68. Ludwig AB, Burton W, Weingarten J, Milan F, Myers DC, Kligler B. Depression and stress amongst undergraduate medical students. BMC Med Educ. 2015;15:141.

69. Park KH, Kim DH, Kim SK, Yi YH, Jeong JH, Chae J, et al. The relationships between empathy, stress and social support among medical students. Int J Med Educ. 2015;6:103-8

70. Dyrbye LN, Harper W, Durning SJ, Moutier C, Thomas MR, Massie FS Jr, et al. Patterns of distress in US medical students. Med Teach. 2011;33(10):834-9.

71. Shapiro J. Walking a mile in their patients' shoes: empathy and othering in medical students' education. Philos Ethics Humanit Med. 2008;3:10.

72. Hojat M, Vergare MJ, Maxwell K, Brainard G, Herrine SK, Isenberg GA, et al. The devil is in the third year: a longitudinal study of erosion of empathy in medical school. Acad Med. 2009;84(9):1182-91.

73. Morse DS, Edwardsen EA, Gordon HS. Missed opportunities for interval empathy in lung cancer communication. Arch Intern Med. 2008;168(17): 1853-8.

74. Kataoka HU, Koide N, Ochi K, Hojat M, Gonnella JS. Measurement of empathy among Japanese medical students: psychometrics and score differences by gender and level of medical education. Acad Med. 2009; 84(9):1192-7.

75. Sulzer SH, Feinstein NW, Wendland CL. Assessing empathy development in medical education: a systematic review. Med Educ. 2016;50(3):300-10.

\section{Publisher's Note}

Springer Nature remains neutral with regard to jurisdictional claims in published maps and institutional affiliations. 